\title{
REISSNER-MINDLIN PLATE THEORY FOR ELASTODYNAMICS
}

\author{
SHEN RONG WU
}

Received 14 January 2004 and in revised form 11 May 2004

Existence and uniqueness of solution are proved for elastodynamics of Reissner-Mindlin plate model. Higher regularity is proved under the assumptions of smoother data and certain compatibility conditions. A mass scaling is introduced. When the thickness approaches zero, the solution of the clamped Reissner-Mindlin plate is shown to approach the solution of a Kirchhoff-Love plate.

\section{Introduction}

The Reissner-Mindlin (R-M) theory $[13,14,15]$ has been popularly applied to thinwalled structures with moderate thickness. Transient response plays an important role in many aspects of structural analysis. The governing equation of the elastodynamics problem of R-M plate is of an evolutionary type with second-order time derivatives. In this paper, we apply a priori estimate to investigate the elastodynamics problem of R-M plate. This method has been successfully used in developing the theory of various partial differential equations, for example, $[7,10,11]$. Following the line of $[7,10,11]$, we prove the existence and uniqueness of the $H^{1}$ solution. We then apply the approaches of [8] to prove the $H^{2}$ regularity and higher regularity when the data is smoother and certain compatibility conditions are satisfied.

For static problem under the assumption of load scaling, it is proved in [3] that the solution of the clamped R-M plate approaches the solution of the Kirchhoff-Love (K-L) plate when the thickness approaches zero. This fact has been employed to investigate the finite element method of R-M plate, such as locking-free and uniform convergence, cf. $[2,3,5,12,16,18]$. For dynamics problem, with the introduction of mass scaling [4], we prove that when thickness approaches zero, the $H^{2}$ strong solution of the clamped R-M plate approaches the $H^{2}$ weak solution of K-L plate (whose classical solution requires $H^{4}$ smoothness).

In what follows, we describe the system of equations in Section 2 and prove the existence, uniqueness, and regularity in Section 3. Then we discuss the relation between R-M plate and K-L plate in Section 4. This is followed by a summary in Section 5. 


\section{Governing equations of Reissener-Mindlin plate for elastodynamics}

For elastodynamic bending shear problem modeled by R-M plate theory $[13,14,15]$, the displacement components of a generic point at a distance $z$ to the midsurface are expressed by the deflection $w$ at the midsurface and the rotations $\left(\beta_{1}, \beta_{2}\right)$ of the normal to the midsurface,

$$
U_{1}=-z \beta_{1}, \quad U_{2}=-z \beta_{2}, \quad U_{3}=w,
$$

$|z| \leq \zeta / 2$. $\zeta$ is the thickness of the plate. For dynamics problems, the velocity and acceleration, traditionally denoted by $\dot{U}_{i}$ and $\ddot{U}_{i}$, respectively, have the same format of $(2.1)$ after differentiation with respect to time. The motion equation of R-M plate can be derived from the general three-dimensional elastodynamics by integration through thickness, or from the energy method using Hamilton's principle, for example, [9]

$$
\begin{gathered}
I \ddot{\beta}_{1}+E A_{1}(\boldsymbol{\beta})-\lambda \zeta^{-2}\left(w,,_{1}-\beta_{1}\right)=m_{1}, \\
I \ddot{\beta}_{2}+E A_{2}(\boldsymbol{\beta})-\lambda \zeta^{-2}\left(w, 2-\beta_{2}\right)=m_{2}, \\
\rho \zeta^{-2} \ddot{w}-\lambda \zeta^{-2} \nabla \cdot(\nabla w-\boldsymbol{\beta})=g=f_{3} \zeta^{-2},
\end{gathered}
$$

where we define

$$
\begin{aligned}
& A_{1}(\boldsymbol{\beta})=\frac{-\left((1+\nu)\left(\beta_{\alpha, \alpha}\right),_{1}+(1-\nu) \nabla^{2} \beta_{1}\right)}{24\left(1-\nu^{2}\right)}, \\
& A_{2}(\boldsymbol{\beta})=\frac{-\left((1+\nu)\left(\beta_{\alpha, \alpha}\right),_{2}+(1-\nu) \nabla^{2} \beta_{2}\right)}{24\left(1-\nu^{2}\right)} .
\end{aligned}
$$

Here, $E$ is the Young's modulus, $\rho$ is the density, and $v$ is the Poisson ratio. We denote $I=\rho / 12$ and $\lambda=G \kappa$, with the shear modulus $G$ and a shear correction factor $\kappa$, which is introduced to balance the zero shear stress at the top and bottom surfaces. As analyzed for static problem $[2,3,5]$, the lateral loading force $f_{3}$ (per unit volume) is scaled to $\zeta^{2} g$. The convention of summation on repeated indices is also applied, with the Greek index running over the range from 1 to 2 . The bold-faced variables are used to denote a twodimensional vector, for example, $\boldsymbol{\beta}=\left(\beta_{1}, \beta_{2}\right)$, and $\beta_{\alpha}$ is used to indicate all of the two components involved when the indication is clear. Here, $w_{1}$ in (2.2) indicates the partial derivative $\partial w / \partial x_{1}$. The same applies for all the similar cases.

For simplicity, we consider the equations defined on a smooth bounded domain $\Omega$ in $R^{2}$, with homogeneous Dirichlet boundary conditions and general initial conditions

$$
\begin{aligned}
& \beta_{\alpha}(t, \mathbf{x})=0 ; \quad w(t, \mathbf{x})=0 \quad \text { on } \partial \Omega, \\
& \beta_{\alpha}(0, \mathbf{x})=\beta_{\alpha}^{0}(\mathbf{x}) ; \quad w(0, \mathbf{x})=W^{0}(\mathbf{x}), \\
& \dot{\beta}_{\alpha}(0, \mathbf{x})=\beta_{\alpha}^{1}(\mathbf{x}) ; \quad \dot{w}(0, \mathbf{x})=W^{1}(\mathbf{x}) .
\end{aligned}
$$


We adopt the usual notations of Sobolev spaces. The Galerkin method yields the following variational equation. For any $t \in[0, T]$, find $\beta_{\alpha}, w \in V=H_{0}^{1}(\Omega)$ such that

$$
I\left\langle\ddot{\beta}_{\alpha}, \eta_{\alpha}\right\rangle+\rho \zeta^{-2}\langle\ddot{w}, \nu\rangle+E a(\boldsymbol{\beta}, \boldsymbol{\eta})+\lambda \zeta^{-2}\left(w_{\alpha}-\beta_{\alpha}, \nu_{\alpha \alpha}-\eta_{\alpha}\right)=\left(m_{\alpha}, \eta_{\alpha}\right)+(g, \nu), \quad \forall \eta_{\alpha}, \nu \in V .
$$

Here, $(\cdot, \cdot)$ denotes the usual $L^{2}$ inner product, and $\langle\cdot, \cdot \cdot\rangle$ denotes the duality on $V^{\prime} \otimes V$. $a(\cdot, \cdot)$ is a bilinear form on $V \otimes V$ defined as

$$
a(\boldsymbol{\beta}, \boldsymbol{\eta})=\frac{1}{24\left(1-\nu^{2}\right)}\left((1+\nu)\left(\beta_{\alpha, \alpha}, \eta_{\alpha, \alpha}\right)+(1-\nu)\left(\nabla \beta_{\alpha}, \nabla \eta_{\alpha}\right)\right) .
$$

It is associated with the operators $A_{1}$ and $A_{2}$ such that

$$
a(\boldsymbol{\beta}, \boldsymbol{\eta})=\left\langle A_{1}(\boldsymbol{\beta}), \eta_{1}\right\rangle+\left\langle A_{2}(\boldsymbol{\beta}), \eta_{2}\right\rangle=\langle\mathbf{A}(\boldsymbol{\beta}), \boldsymbol{\eta}\rangle, \quad \forall \beta_{\alpha}, \eta_{\alpha} \in V .
$$

In fact, $a(\cdot, \cdot)$ is symmetric, the same as the two-dimensional elasticity operator with a scalar factor. With Dirichlet boundary conditions, $a(\cdot, \cdot)$ is equivalent to the $H^{1}$-norm on $V[6]$ and there exist constants $\alpha_{1}, \alpha_{2}>0$ such that

$$
\begin{gathered}
\alpha_{1}\|\boldsymbol{\eta}\|_{1}^{2} \leq a(\boldsymbol{\eta}, \boldsymbol{\eta}), \quad \forall \eta_{\alpha} \in V, \\
a(\boldsymbol{\beta}, \boldsymbol{\eta}) \leq \alpha_{2}\|\boldsymbol{\beta}\|_{1}\|\boldsymbol{\eta}\|_{1}, \quad \forall \beta_{\alpha,} \eta_{\alpha} \in V .
\end{gathered}
$$

We denote $\|\boldsymbol{\eta}\|_{x}^{2}=\left\|\eta_{1}\right\|_{x}^{2}+\left\|\eta_{2}\right\|_{x}^{2}$ for $\eta_{\alpha}$ of a functional space $X$. Note that for timedependent problems, the norm $\|\nu\|_{X}$ of a function $\nu:[0, T] \rightarrow X$ is a function of time. We use the following notations for the functional spaces and the measure in time:

$$
\begin{aligned}
L^{2}(X) & =L^{2}(0, T ; X) \\
& =\left\{v:[0, T] \longrightarrow X \mid v(t, \mathbf{x}) \in X,\|v\|_{L^{2}(0, T ; X)}=\left(\int_{0}^{T}\left(\|v\|_{X}\right)^{2} d t\right)^{1 / 2}<\infty\right\}, \\
L^{\infty}(X) & =L^{\infty}(0, T ; X) \\
& =\left\{v:[0, T] \longrightarrow X \mid v(t, \mathbf{x}) \in X,\|\nu\|_{L^{\infty}(0, T ; X)}=\underset{0 \leq t \leq T}{\operatorname{esssup}}\left(\|v\|_{X}\right)<\infty\right\} .
\end{aligned}
$$

\section{Existence, uniqueness, and regularity}

For linear hyperbolic equations of the second order in time with one function, the existence and uniqueness are proven (see, e.g., $[7,10,11]$ ) using the method of a priori estimate. The method is also employed in [17] for Navier-Stokes problem whose steadystate case has close relation to the static problems of R-M plate, (cf. [5]). We extend the scheme to the dynamic problems of R-M plate. For the time being, we keep the material parameters explicitly expressed for later use in Section 4.

Theorem 3.1. If $m_{\alpha}, g \in L^{2}\left(L^{2}\right) ; B_{\alpha}^{0}, W^{0} \in H_{0}^{1}$; and $B_{\alpha}^{1}, W^{1} \in L^{2}$, then there exists a solution $\left(\beta_{\alpha}, w\right)$ of (2.5) (a weak solution of (2.2)) with initial conditions (2.4), $\beta_{\alpha}, w \in L^{\infty}\left(H_{0}^{1}\right)$, $\dot{\beta}_{\alpha}, \dot{w} \in L^{\infty}\left(L^{2}\right)$, and $\ddot{\beta}_{\alpha}, \ddot{w} \in L^{\infty}\left(H^{-1}\right)$. Moreover, there exists a constant $C>0$, independent 
182 Reissner-Mindlin plate theory for elastodynamics

of the material parameters, such that

$$
\begin{aligned}
& I\|\dot{\boldsymbol{\beta}}\|_{0}^{2}+\rho \zeta^{-2}\|\dot{w}\|_{0}^{2}+E\|\boldsymbol{\beta}\|_{1}^{2}+\lambda \zeta^{-2}\|\nabla w-\boldsymbol{\beta}\|_{0}^{2} \\
& \leq C\left(I\left\|\mathbf{B}^{1}\right\|_{0}^{2}+\rho \zeta^{-2}\left\|W^{1}\right\|_{0}^{2}+E\left\|\mathbf{B}^{0}\right\|_{1}^{2}\right. \\
& \left.\quad+\lambda \zeta^{-2}\left\|\nabla W^{0}-\mathbf{B}^{0}\right\|_{0}^{2}+I^{-1}\|\mathbf{m}\|_{L^{2}\left(L^{2}\right)}^{2}+\rho^{-1} \zeta^{2}\|g\|_{L^{2}\left(L^{2}\right)}^{2}\right)
\end{aligned}
$$

Proof. We apply the scheme for hyperbolic equations developed in $[7,10,11]$. The space $V$ is separable. We construct an approximation of order $n$, with a countable basis $\left\{\psi_{i}(\mathbf{x})\right.$, $i=1,2, \ldots\}$ of $V$ :

$$
\begin{gathered}
\beta_{\alpha n}(t, \mathbf{X})=\sum_{j=1}^{n} \beta_{\alpha n}^{j}(t) \psi_{j}(\mathbf{X}), \quad w_{n}(t, \mathbf{X})=\sum_{j=1}^{n} w_{n}^{j}(t) \psi_{j}(\mathbf{X}), \\
I\left(\ddot{\beta}_{\alpha n}, \eta_{\alpha}\right)+\rho \zeta^{-2}\left(\ddot{w}_{n}, \nu\right)+E a\left(\boldsymbol{\beta}_{n}, \boldsymbol{\eta}\right)+\lambda \zeta^{-2}\left(w_{n, \alpha}-\beta_{\alpha n}, \nu_{\alpha}-\eta_{\alpha}\right) \\
=(g, \nu)+\left(m_{\alpha}, \eta_{\alpha}\right), \quad \forall \eta_{\alpha}, \nu \in V_{n}=\operatorname{span}\left\{\psi_{1}, \ldots, \psi_{n}\right\} .
\end{gathered}
$$

The approximation problem (3.2) leads to a linear system of second-order ordinary differential equations. With the approximations of (2.4) for the initial conditions

$$
\begin{aligned}
& \sum_{j=1}^{n} B_{\alpha n}^{0 j} \psi_{j}(\mathbf{X})=B_{\alpha n}^{0} \longrightarrow B_{\alpha}^{0}, \quad \sum_{j=1}^{n} W_{n}^{0 j} \psi_{j}(\mathbf{X})=W_{n}^{0} \longrightarrow W^{0}, \\
& \sum_{j=1}^{n} B_{\alpha n}^{1 j} \psi_{j}(\mathbf{X})=B_{\alpha n}^{1} \longrightarrow B_{\alpha}^{1}, \quad \sum_{j=1}^{n} W_{n}^{1 j} \psi_{j}(\mathbf{X})=W_{n}^{1} \longrightarrow W^{1},
\end{aligned}
$$

we have unique solution

$$
\left\{\beta_{1 n}^{j}(t), \beta_{2 n}^{j}(t), w_{n}^{j}(t), j=1, \ldots, n\right\} \in H^{2}([0, T]) .
$$

Now using $\eta_{\alpha}=\dot{\beta}_{\alpha n}(t, \mathbf{X})$ and $\nu=\dot{w}_{n}(t, \mathbf{X})$ in (3.2), then integrating from $t=0$ to $T$, we have

$$
\begin{aligned}
I\left\|\dot{\boldsymbol{\beta}}_{n}\right\|_{0}^{2} & +\rho \zeta^{-2}\left\|\dot{w}_{n}\right\|_{0}^{2}+E a\left(\boldsymbol{\beta}_{n}, \boldsymbol{\beta}_{n}\right)+\lambda \zeta^{-2}\left\|\nabla w_{n}-\boldsymbol{\beta}_{n}\right\|_{0}^{2} \\
= & I\left\|\dot{\boldsymbol{\beta}}_{n}(0)\right\|_{0}^{2}+\rho \zeta^{-2}\left\|\dot{w}_{n}(0)\right\|_{0}^{2}+E a\left(\boldsymbol{\beta}_{n}(0), \boldsymbol{\beta}_{n}(0)\right) \\
& +\lambda \zeta^{-2}\left\|\nabla w_{n}(0)-\boldsymbol{\beta}_{n}(0)\right\|_{0}^{2}+2 \int_{0}^{T}\left(\left(m_{\alpha}, \dot{\beta}_{\alpha n}\right)+\left(g, \dot{w}_{n}\right)\right) d t \\
\leq & I\left\|\mathbf{B}_{n}^{1}\right\|_{0}^{2}+\rho \zeta^{-2}\left\|W_{n}^{1}\right\|_{0}^{2}+E \alpha_{2}\left\|\mathbf{B}_{n}^{0}\right\|_{1}^{2}+\lambda \zeta^{-2}\left\|\nabla W_{n}^{0}-\mathbf{B}_{n}^{0}\right\|_{0}^{2} \\
& +\int_{0}^{T}\left(I^{-1}\|\mathbf{m}\|_{0}^{2}+\rho^{-1} \zeta^{2}\|g\|_{0}^{2}\right) d t+\int_{0}^{T}\left(I\left\|\dot{\boldsymbol{\beta}}_{n}\right\|_{0}^{2}+\rho \zeta^{-2}\left\|\dot{w}_{n}\right\|_{0}^{2}\right) d t .
\end{aligned}
$$


Applying Gronwall inequality, we obtain

$$
\begin{aligned}
& I\left\|\dot{\boldsymbol{\beta}}_{n}\right\|_{0}^{2}+\rho \zeta^{-2}\left\|\dot{w}_{n}\right\|_{0}^{2}+\alpha_{1} E\left\|\boldsymbol{\beta}_{n}\right\|_{1}^{2}+\lambda \zeta^{-2}\left\|\nabla w_{n}-\boldsymbol{\beta}_{n}\right\|_{0}^{2} \\
& \leq C\left(I\left\|\mathbf{B}_{n}^{1}\right\|_{0}^{2}+\rho \zeta^{-2}\left\|W_{n}^{1}\right\|_{0}^{2}+\alpha_{2} E\left\|\mathbf{B}_{n}^{0}\right\|_{1}^{2}\right. \\
& \left.\quad+\lambda \zeta^{-2}\left\|\nabla W_{n}^{0}-\mathbf{B}_{n}^{0}\right\|_{0}^{2}+I^{-1}\|\mathbf{m}\|_{L^{2}\left(L^{2}\right)}^{2}+\rho^{-1} \zeta^{2}\|g\|_{L^{2}\left(L^{2}\right)}^{2}\right) .
\end{aligned}
$$

The right-hand side has limit as $n \rightarrow \infty$ due to (3.3). Therefore, the left-hand side is bounded. Note that $\left\|w_{n}\right\|_{1} \leq C\left\|\nabla w_{n}\right\|_{0} \leq C\left(\left\|\nabla w_{n}-\boldsymbol{\beta}_{n}\right\|_{0}+\left\|\boldsymbol{\beta}_{n}\right\|_{0}\right)$. By compactness, we can find convergent subsequences, still denoted by subscript $n$, such that

$$
\begin{array}{lll}
\beta_{\alpha n} \longrightarrow \beta_{\alpha}, & w_{n} \longrightarrow w & \text { weakly star in } L^{\infty}\left(H_{0}^{1}\right), \\
\dot{\beta}_{\alpha n} \longrightarrow \varphi_{\alpha}, & \dot{w}_{n} \longrightarrow \chi & \text { weakly star in } L^{\infty}\left(L^{2}\right) .
\end{array}
$$

It is a straightforward task to verify that $\dot{\beta}_{\alpha}=\varphi_{\alpha}, \dot{w}=\chi, \ddot{\beta}_{\alpha n} \rightarrow \ddot{\beta}_{\alpha}, \ddot{w}_{n} \rightarrow \ddot{w}$ weakly star in $L^{\infty}\left(H^{-1}\right)$, and $\left\{\beta_{\alpha}, w\right\}$ satisfy the initial conditions (2.4) and the variational equation (2.5), thus form a weak solution of (2.2).

Theorem 3.2. Under the conditions of Theorem 3.1, the solution $\left\{\beta_{\alpha}, w\right\}$ is unique, that is, if $g=0, m_{\alpha}=0, B_{\alpha}^{0}=w^{0}=B_{\alpha}^{1}=w^{1}=0$, then $\beta_{\alpha}=w=0$.

Proof. Following the line of $[10,11]$, we can prove the uniqueness, but omit the details.

Theorem 3.3. Under the conditions of Theorem 3.1, if $\dot{m}_{\alpha}, \dot{g} \in L^{2}\left(L^{2}\right), B_{\alpha}^{0}, W^{0} \in H^{2}$, and $B_{\alpha}^{1}, W^{1} \in H_{0}^{1}$, then the solution $\left(\beta_{\alpha}, w\right)$ of (2.2) with the initial conditions (2.4) satisfies $\ddot{\beta}_{\alpha}, \ddot{w} \in L^{\infty}\left(L^{2}\right), \dot{\beta}_{\alpha}, \dot{w} \in L^{\infty}\left(H_{0}^{1}\right), \beta_{\alpha}, w \in L^{\infty}\left(H^{2}\right)$, and

$$
\begin{gathered}
I\|\ddot{\boldsymbol{\beta}}\|_{0}^{2}+\rho \zeta^{-2}\|\ddot{w}\|_{0}^{2}+E\|\dot{\boldsymbol{\beta}}\|_{1}^{2}+\zeta^{-2}\|\nabla \dot{\boldsymbol{w}}-\dot{\boldsymbol{\beta}}\|_{0}^{2} \\
\leq C\left(I^{-1}\left(E^{2}\left\|\mathbf{B}^{0}\right\|_{2}^{2}+\lambda^{2} \zeta^{-4}\left\|\nabla W^{0}-\mathbf{B}^{0}\right\|_{0}^{2}+\|\mathbf{m}(0)\|_{0}^{2}\right)\right. \\
+\rho^{-1} \zeta^{2}\left(\lambda^{2} \zeta^{-4}\left\|\nabla W^{0}-\mathbf{B}^{0}\right\|_{1}^{2}+\|g(0)\|_{0}^{2}\right) \\
\left.+E\left\|\mathbf{B}^{1}\right\|_{1}^{2}+\lambda \zeta^{-2}\left\|\nabla W^{1}-\mathbf{B}^{1}\right\|_{0}^{2}+I^{-1}\|\dot{\mathbf{m}}\|_{L^{2}\left(L^{2}\right)}^{2}+\rho^{-1} \zeta^{2}\|\dot{g}\|_{L^{2}\left(L^{2}\right)}^{2}\right), \\
E\|\boldsymbol{\beta}\|_{2} \leq C\left(I\|\ddot{\boldsymbol{\beta}}\|_{0}+\lambda \zeta^{-2}\|\nabla w-\boldsymbol{\beta}\|_{0}+\|\mathbf{m}\|_{0}\right), \\
\lambda \zeta^{-2}\|w\|_{2} \leq C\left(\rho \zeta^{-2}\|\ddot{w}\|_{0}+\lambda \zeta^{-2}\|\beta\|_{1}+\|g\|_{0}\right),
\end{gathered}
$$

where the bounds of $\|\nabla w-\beta\|_{0}$ and $\|\beta\|_{1}$ are established in (3.1).

Proof. We apply the method for hyperbolic equations demonstrated in [8]. From Theorem 3.1, we have $\dot{\beta}_{\alpha n}^{j}, \dot{w}_{n}^{j} \in H^{1}([0, T])$. Differentiating (3.2) with respect to $t$, we obtain $\dddot{\beta}_{\alpha n}^{j}, \dddot{w}_{n}^{j} \in L^{2}([0, T])$. The a priori estimate like (3.6) holds:

$$
\begin{aligned}
& I\left\|\ddot{\boldsymbol{\beta}}_{n}\right\|_{0}^{2}+\rho \zeta^{-2}\left\|\ddot{w}_{n}\right\|_{0}^{2}+\alpha_{1} E\left\|\dot{\boldsymbol{\beta}}_{n}\right\|_{1}^{2}+\lambda \zeta^{-2}\left\|\nabla \dot{w}_{n}-\dot{\boldsymbol{\beta}}_{n}\right\|_{0}^{2} \\
& \leq C\left(I\left\|\ddot{\boldsymbol{\beta}}_{n}(0)\right\|_{0}^{2}+\rho \zeta^{-2}\left\|\ddot{w}_{n}(0)\right\|_{0}^{2}+\alpha_{2} E\left\|\dot{\boldsymbol{\beta}}_{n}(0)\right\|_{1}^{2}\right. \\
& \left.\quad+\lambda \zeta^{-2}\left\|\nabla \dot{w}_{n}(0)-\dot{\boldsymbol{\beta}}_{n}(0)\right\|_{0}^{2}+I^{-1}\|\dot{\mathbf{m}}\|_{L^{2}\left(L^{2}\right)}^{2}+\rho^{-1} \zeta^{2}\|\dot{g}\|_{L^{2}\left(L^{2}\right)}^{2}\right) .
\end{aligned}
$$


184 Reissner-Mindlin plate theory for elastodynamics

From $(2.2)$, we obtain $\ddot{\beta}_{\alpha}(0), \ddot{w}(0) \in L^{2}$,

$$
\begin{gathered}
I\left\|\ddot{\beta}_{\alpha}(0)\right\|_{0} \leq E\left\|\mathbf{B}^{0}\right\|_{2}+\lambda \zeta^{-2}\left\|\nabla W^{0}-\mathbf{B}^{0}\right\|_{0}+\left\|m_{\alpha}(0)\right\|_{0}, \\
\rho \zeta^{-2}\|\ddot{w}(0)\|_{0} \leq \lambda \zeta^{-2}\left\|\nabla W^{0}-\mathbf{B}^{0}\right\|_{1}+\|g(0)\|_{0} .
\end{gathered}
$$

The argument of boundedness and compactness leads to the conclusion that $\ddot{\beta}_{\alpha n} \rightarrow \ddot{\beta}_{\alpha}$, $\ddot{w}_{n} \rightarrow \ddot{w}$ weakly star in $L^{\infty}\left(L^{2}\right)$. Hence, (3.11) implies (3.8).

On the other hand, we rewrite (2.2):

$$
\begin{aligned}
E A_{1}(\boldsymbol{\beta}) & =m_{1}-I \ddot{\beta}_{1}+\lambda \zeta^{-2}\left(w,_{1}-\beta_{1}\right), \\
E A_{2}(\boldsymbol{\beta}) & =m_{2}-I \ddot{\beta}_{2}+\lambda \zeta^{-2}\left(w, 2-\beta_{2}\right), \\
-\lambda \zeta^{-2} \nabla^{2} w & =g-\rho \zeta^{-2} \ddot{w}-\lambda \zeta^{-2} \beta_{\alpha, \alpha} .
\end{aligned}
$$

For any fixed time $t$, the right-hand sides of these equations are in $L^{2}$. We have the elasticity operator and the Laplace operator in the left-hand side. According to the theory of elliptic equations, with a smooth domain $\Omega$, we have $\beta_{\alpha}, w \in H^{2}$, and the bounds (3.9) and (3.10).

We are ready to extend the method for higher regularity of hyperbolic equation [8] to the transient dynamics of R-M plate. For simplicity, the dependence on the material parameters is not explicitly expressed and will have more discussion in Section 4.

Theorem 3.4. Assume for any integer $P \geq 0$,

$$
\begin{gathered}
B_{\alpha}^{0}, W^{0} \in H^{P+1} \cap H_{0}^{1}, \quad B_{\alpha}^{1}, W^{1} \in H^{P} \cap H_{0}^{1}, \\
\frac{\partial^{k} m_{\alpha}}{\partial t^{k}}, \quad \frac{\partial^{k} g}{\partial t^{k}} \in L^{2}\left(H^{P-k}\right), \quad k=0,1, \ldots, P,
\end{gathered}
$$

and that the following compatibility conditions hold for $P \geq 2$ :

$$
\begin{aligned}
B_{\alpha}^{k+2} & =I^{-1}\left(\frac{\partial^{k} m_{\alpha}(0)}{\partial t^{k}}-E A_{\alpha}\left(\mathbf{B}^{k}\right)+\lambda \zeta^{-2}\left(\nabla W^{k}-\mathbf{B}^{k}\right)\right) \in H_{0}^{1}, \\
W^{k+2} & =\left(\rho \zeta^{-2}\right)^{-1}\left(\frac{\partial^{k} g(0)}{\partial t^{k}}+\lambda \zeta^{-2} \nabla \cdot\left(\nabla W^{k}-\mathbf{B}^{k}\right)\right) \in H_{0}^{1},
\end{aligned}
$$

Then the solution of (2.2) with (2.4) satisfy, for $k=0,1, \ldots, P+1$,

$$
\begin{gathered}
\frac{\partial^{k} \beta_{\alpha}}{\partial t^{k}}, \quad \frac{\partial^{k} w}{\partial t^{k}} \in L^{\infty}\left(H^{P+1-k}\right) \\
\left\|\frac{\partial^{k} \beta_{\alpha}}{\partial t^{k}}\right\|_{P+1-k}+\left\|\frac{\partial^{k} w}{\partial t^{k}}\right\|_{P+1-k} \\
\leq C\left(\sum_{j=0}^{P}\left(\left\|\frac{\partial^{j} m_{\alpha}}{\partial t^{j}}\right\|_{L^{2}\left(H^{P-j}\right)}+\left\|\frac{\partial^{j} g}{\partial t^{j}}\right\|_{L^{2}\left(H^{P-j}\right)}\right)\right. \\
\left.+\left\|\mathbf{B}^{0}\right\|_{P+1}+\left\|W^{0}\right\|_{P+1}+\left\|\mathbf{B}^{1}\right\|_{P}+\left\|W^{1}\right\|_{P}\right) .
\end{gathered}
$$


Proof. The cases of $P=0$ and $P=1$ are proved in Theorems 3.1 and 3.3, respectively. Using the method of induction, we assume that the theorem is true for $P \leq Q$ and assume that the conditions (3.14) and (3.15) are valid for $P=Q+1$. Denote

$$
\begin{gathered}
\tilde{B}_{\alpha}=\dot{\beta}_{\alpha}, \quad \tilde{w}=\dot{w}, \\
\tilde{m}_{\alpha}=\dot{m}_{\alpha}, \quad \tilde{g}=\dot{g}, \\
\tilde{B}_{\alpha}^{k}=B_{\alpha}^{k+1}, \quad \tilde{W}^{k}=W^{k+1}, \quad k=0,1, \ldots, Q .
\end{gathered}
$$

Then $\tilde{B}_{\alpha}^{k}, \tilde{W}^{k}, k=0,1, \ldots, Q$, satisfy (3.15) for $P=Q$. $\tilde{B}_{\alpha}^{0}=B_{\alpha}^{1}$ and $\tilde{W}^{0}=W^{1} \in H^{Q+1} \cap$ $H_{0}^{1}$. For $k=0,1, \ldots, Q, \partial^{k} \tilde{m}_{\alpha} / \partial t^{k}=\partial^{k+1} m_{\alpha} / \partial t^{k+1}$ and $\partial^{k} \tilde{g} / \partial t^{k}=\partial^{k+1} g / \partial t^{k+1} \in L^{2}\left(H^{Q-k}\right)$. From (3.14) and (3.15) with $P=Q+1$,

$$
\begin{aligned}
\left\|\tilde{\mathbf{B}}^{1}\right\|_{Q} & =\left\|\mathbf{B}^{2}\right\|_{Q} \leq C\left(\|\mathbf{m}(0)\|_{Q}+\left\|\mathbf{B}^{0}\right\|_{Q+2}+\left\|W^{0}\right\|_{Q+1}+\left\|\mathbf{B}^{0}\right\|_{Q}\right) \leq \infty, \\
\left\|\tilde{W}^{1}\right\|_{Q} & =\left\|W^{2}\right\|_{Q} \leq C\left(\|g(0)\|_{Q}+\left\|W^{0}\right\|_{Q+2}+\left\|\mathbf{B}^{0}\right\|_{Q+1}\right) \leq \infty .
\end{aligned}
$$

Hence $\tilde{B}_{\alpha}^{k}, \tilde{W}^{k}, k=0$ and 1 , satisfy (3.14) for $P=Q$. We apply the assumption of induction and obtain from (3.16) and (3.17) with $P=Q$, for $k=0,1, \ldots, Q+1$,

$$
\begin{gathered}
\frac{\partial^{k} \tilde{B}_{\alpha}}{\partial t^{k}}, \quad \frac{\partial^{k} \tilde{w}}{\partial t^{k}} \in L^{\infty}\left(H^{Q+1-k}\right) \\
\left\|\frac{\partial^{k} \tilde{\beta}_{\alpha}}{\partial t^{k}}\right\|_{Q+1-k}+\left\|\frac{\partial^{k} \tilde{w}}{\partial t^{k}}\right\|_{Q+1-k} \\
\leq C\left(\sum_{j=0}^{Q}\left(\left\|\frac{\partial^{j} \stackrel{\leftrightarrow}{m}_{\alpha}}{\partial t^{j}}\right\|_{L^{2}\left(H^{Q-j}\right)}+\left\|\frac{\partial^{j} \tilde{g}}{\partial t^{j}}\right\|_{L^{2}\left(H^{Q-j}\right)}\right)\right. \\
\left.+\left\|\tilde{\mathbf{B}}^{0}\right\|_{Q+1}+\left\|\tilde{W}^{0}\right\|_{Q+1}+\left\|\tilde{\mathbf{B}}^{1}\right\|_{Q}+\left\|\tilde{W}^{1}\right\|_{Q}\right) .
\end{gathered}
$$

It implies that, for $k=1, \ldots, Q+2$,

$$
\begin{gathered}
\frac{\partial^{k} \beta_{\alpha}}{\partial t^{k}}, \quad \frac{\partial^{k} w}{\partial t^{k}} \in L^{\infty}\left(H^{Q+2-k}\right) \\
\left\|\frac{\partial^{k} \beta_{\alpha}}{\partial t^{k}}\right\|_{Q+2-k}+\left\|\frac{\partial^{k} w}{\partial t^{k}}\right\|_{Q+2-k} \\
\leq C\left(\sum_{j=0}^{Q+1}\left(\left\|\frac{\partial^{j} m_{\alpha}}{\partial t^{j}}\right\|_{L^{2}\left(H^{Q+1-j}\right)}+\left\|\frac{\partial^{j} g}{\partial t^{j}}\right\|_{L^{2}\left(H^{Q+1-j}\right)}\right)\right. \\
\left.+\left\|\mathbf{B}^{1}\right\|_{Q+1}+\left\|W^{1}\right\|_{Q+1}+\left\|\mathbf{B}^{2}\right\|_{Q}+\left\|W^{2}\right\|_{Q}\right) .
\end{gathered}
$$

We can use (3.19) to estimate $\mathbf{B}^{2}$ and $W^{2}$ in (3.23) with

$$
\begin{gathered}
\|\mathbf{m}(0)\|_{Q} \leq C\|\mathbf{m}\|_{C^{0}\left(H^{Q}\right)} \leq C\left(\|\mathbf{m}\|_{L^{2}\left(H^{Q}\right)}+\|\dot{\mathbf{m}}\|_{L^{2}\left(H^{Q}\right)}\right), \\
\|g(0)\|_{Q} \leq C\|g\|_{C^{0}\left(H^{Q}\right)} \leq C\left(\|g\|_{L^{2}\left(H^{Q}\right)}+\|\dot{g}\|_{L^{2}\left(H^{Q}\right)}\right) .
\end{gathered}
$$


Therefore, (3.17) is true for $P=Q+1$ and $k=1, \ldots, Q+2$. Now the right-hand sides of (3.13) are bounded in $H^{Q}$. We have

$$
\begin{aligned}
& \|\boldsymbol{\beta}\|_{Q+2}^{2} \leq C\left\|\mathbf{I} \ddot{\boldsymbol{\beta}}-\lambda \zeta^{-2}(\nabla w-\boldsymbol{\beta})-\mathbf{m}\right\|_{Q}^{2} \leq C\left(\|\ddot{\boldsymbol{\beta}}\|_{Q}^{2}+\|w\|_{Q+1}^{2}+\|\boldsymbol{\beta}\|_{Q}^{2}+\|\mathbf{m}\|_{Q}^{2}\right) \leq \infty, \\
& \|w\|_{Q+2}^{2} \leq C\left\|\rho \zeta^{-2} \ddot{w}+\lambda \zeta^{-2} \beta_{\alpha, \alpha}-g\right\|_{Q}^{2} \leq C\left(\|\ddot{w}\|_{Q}^{2}+\|\boldsymbol{\beta}\|_{Q+1}^{2}+\|g\|_{Q}^{2}\right) \leq \infty .
\end{aligned}
$$

Therefore,

$$
\begin{aligned}
\|\boldsymbol{\beta}\|_{Q+2}+\|w\|_{Q+2} \\
\leq C\left(\sum_{j=0}^{Q+1}\left(\left\|\frac{\partial^{j} m_{\alpha}}{\partial t^{j}}\right\|_{L^{2}\left(H^{Q+1-j}\right)}+\left\|\frac{\partial^{j} g}{\partial t^{j}}\right\|_{L^{2}\left(H^{Q+1-j}\right)}\right)\right. \\
\left.+\left\|\mathbf{B}^{0}\right\|_{Q+2}+\left\|W^{0}\right\|_{Q+2}+\left\|\mathbf{B}^{1}\right\|_{Q+1}+\left\|W^{1}\right\|_{Q+1}\right) .
\end{aligned}
$$

Thus, (3.17) also holds for $P=Q+1$ and $k=0$. The case of $P=Q+1$ of the induction is true.

\section{Relation to Kirchhoff-Love plate}

For static problem, it is understood that when the thickness $\zeta \rightarrow 0$, the solution of the clamped R-M plate approaches the solution of a K-L plate (see, e.g., [3] for a proof). The convergence is for the systems with load scaling, in the sense that $\beta_{\alpha} \rightarrow \tilde{\beta}_{\alpha}, w \rightarrow \tilde{w}$, and

$$
\begin{gathered}
\tilde{\boldsymbol{\beta}}=\nabla \tilde{\boldsymbol{w}}, \\
D_{0} \nabla^{4} \tilde{\boldsymbol{w}}=g,
\end{gathered}
$$

where $D_{0}=E / 12\left(1-v^{2}\right)=D \zeta^{-3}$. $D$ is the usual bending stiffness. Due to the load scaling, the K-L equation (4.2) is independent of thickness. Physically, when the thickness approaches zero, the bending stiffness approaches zero faster with a factor of $\zeta^{3}$. The unscaled loading, which contributes to the external work, is proportional to the thickness and will not give a meaningful solution. This fact is used for investigating the thicknessindependent convergence of finite element method, for example, $[2,3,5,12,16,18]$ (see $[12,16,18]$ for numerical examples).

For dynamic problem, due to the appearance of the inertia term, which contributes to the kinetic energy, the equation of K-L plate is no longer thickness independent. To keep $\mathrm{K}-\mathrm{L}$ plate as a reference model, a possible approach is then to scale the mass density [4] along with the load. Assume

$$
\begin{gathered}
\rho=\zeta^{2} \rho_{0}, \\
I=\zeta^{2} I_{0}, \quad I_{0}=\frac{\rho_{0}}{12} .
\end{gathered}
$$


We consider the scaled R-M equation (2.2) with $m_{\alpha}=0$, which does not appear in K-L plate:

$$
\begin{gathered}
I_{0} \zeta^{2} \ddot{\beta}_{1}+E A_{1}(\boldsymbol{\beta})-\lambda \zeta^{-2}\left(w,,_{1}-\beta_{1}\right)=0, \\
I_{0} \zeta^{2} \ddot{\beta}_{2}+E A_{2}(\boldsymbol{\beta})-\lambda \zeta^{-2}\left(w, 2-\beta_{2}\right)=0, \\
\rho_{0} \ddot{w}-\lambda \zeta^{-2} \nabla \cdot(\nabla w-\boldsymbol{\beta})=g,
\end{gathered}
$$

or the variational equation (2.5):

$$
I_{0} \zeta^{2}\left\langle\ddot{\beta}_{\alpha}, \eta_{\alpha}\right\rangle+\rho_{0}\langle\ddot{w}, \nu\rangle+E a(\boldsymbol{\beta}, \boldsymbol{\eta})+\lambda \zeta^{-2}\left(w,_{\alpha}-\beta_{\alpha}, \nu_{\alpha}-\eta_{\alpha}\right)=(g, \nu), \quad \forall \eta_{\alpha}, \nu \in V .
$$

As a parallel study to the static problem, we consider a special case of elastodynamics with zero initial conditions:

$$
B_{\alpha}^{0}=B_{\alpha}^{1}=W^{0}=W^{1}=0 .
$$

Theorem 4.1. Assume $g \in H^{1}\left(L^{2}\right), \dot{g} \in L^{2}\left(L^{2}\right)$, and $\left(\beta_{\alpha}, w\right) \in L^{\infty}\left(H_{0}^{1}\right)$ is the solution of (4.4) (or (4.5)) with initial conditions (4.6). Then as $\zeta \rightarrow 0$, there exists a sequence of $\left(\beta_{\alpha}, w\right)$ with the same notation for simplicity such that

$$
\begin{aligned}
& w \longrightarrow \tilde{w} \quad \text { weaklystar in } L^{\infty}\left(H^{2}\right), \\
& \beta_{\alpha} \longrightarrow \tilde{\beta}, \quad \dot{w} \longrightarrow \dot{\tilde{w}} \quad \text { weakly star in } L^{\infty}\left(H^{1}\right), \\
& \ddot{w} \longrightarrow \ddot{\tilde{w}} \quad \text { weakly star in } L^{\infty}\left(L^{2}\right) .
\end{aligned}
$$

Moreover,

$$
\tilde{\boldsymbol{\beta}}=\nabla \tilde{w}
$$

and $\tilde{w}$ is the solution of a K-L plate problem of elastodynamics with clamped boundary conditions

$$
\begin{gathered}
\rho_{0} \ddot{\tilde{\tilde{w}}}+D_{0} \nabla^{4} \tilde{\mathcal{w}}=g \quad \text { or } \\
\rho_{0}(\ddot{\tilde{w}}, v)+D_{0}\left(\nabla^{2} \tilde{w}, \nabla^{2} v\right)=(g, v), \quad \forall v \in H_{0}^{2}, \\
\left.\tilde{w}\right|_{\partial \Omega}=\left.\frac{\partial \tilde{w}}{\partial n}\right|_{\partial \Omega}=0, \\
\tilde{w}(0, \mathbf{x})=\dot{\tilde{w}}(0, \mathbf{x})=0 .
\end{gathered}
$$

Proof. By Theorems 3.1 to 3.3, we have a unique solution $\left(\beta_{\alpha}, w\right) \in L^{\infty}\left(H_{0}^{1}\right) \cap L^{\infty}\left(H^{2}\right)$ for (4.4) (or (4.5)), where the generic constant $C>0$ used in the a priori estimates is independent of material parameters. With (4.6), the a priori estimate (3.1) is reduced to

$$
\sqrt{I_{0}} \zeta\|\dot{\boldsymbol{\beta}}\|_{0}+\sqrt{\rho_{0}}\|\dot{w}\|_{0}+\sqrt{E}\|\boldsymbol{\beta}\|_{1}+\sqrt{\lambda \zeta^{-2}}\|\nabla w-\boldsymbol{\beta}\|_{0} \leq C\|g\|_{L^{2}\left(L^{2}\right)}
$$

Inequality (3.8) yields

$$
\sqrt{I_{0}} \zeta\|\ddot{\boldsymbol{\beta}}\|_{0}+\sqrt{\rho_{0}}\|\ddot{w}\|_{0}+\sqrt{E}\|\dot{\boldsymbol{\beta}}\|_{1}+\sqrt{\lambda \zeta^{-2}}\|\nabla \dot{w}-\dot{\boldsymbol{\beta}}\|_{0} \leq C\left(\|g(0)\|_{0}+\|\dot{g}\|_{L^{2}\left(L^{2}\right)}\right) .
$$


Inequality (3.10) results in

$$
\|w\|_{2} \leq C\left(\|g(0)\|_{0}+\|\dot{g}\|_{L^{2}\left(L^{2}\right)}+\|g\|_{L^{2}\left(L^{2}\right)}\right) .
$$

Furthermore, $\|\dot{w}\|_{1} \leq C\left(\|\dot{w}\|_{0}+\|\nabla \dot{w}\|_{0}\right) \leq C\left(\|\dot{w}\|_{0}+\|\nabla \dot{w}-\dot{\boldsymbol{\beta}}\|_{0}+\|\dot{\boldsymbol{\beta}}\|_{0}\right)$. Therefore, the boundedness of $w$ in $L^{\infty}\left(H^{2}\right), \beta_{\alpha}, \dot{\beta}_{\alpha}, \dot{w}$ in $L^{\infty}\left(H^{1}\right)$, and $\zeta \ddot{\beta}_{\alpha}, \ddot{w}$ in $L^{\infty}\left(L^{2}\right)$ are all uniform with respect to the thickness. We can extract the convergent sequences

$$
\begin{aligned}
& w \longrightarrow \tilde{w} \quad \text { weakly star in } L^{\infty}\left(H^{2}\right), \\
& \beta_{\alpha} \longrightarrow \tilde{\beta}_{\alpha}, \quad \dot{\beta}_{\alpha} \longrightarrow \dot{\tilde{\beta}}_{\alpha}, \quad \dot{w} \longrightarrow \dot{\tilde{w}} \quad \text { weakly star in } L^{\infty}\left(H^{1}\right), \\
& \ddot{w} \longrightarrow \tilde{\tilde{w}} \quad \text { weakly star in } L^{\infty}\left(L^{2}\right),
\end{aligned}
$$

where, for simplicity, no $\zeta$-dependence notation is used for the sequences. The relation with time differentiation is trivial.

The initial conditions $\tilde{w}(0, \mathbf{x})=\dot{\tilde{w}}(0, \mathbf{x})=0$ are a direct result of (4.6). Inequality (4.10) implies $\sqrt{\lambda}\|\nabla w-\boldsymbol{\beta}\|_{0} \leq C \zeta\|g\|_{L^{2}\left(L^{2}\right)} \rightarrow 0 \Rightarrow \nabla \tilde{w}-\tilde{\boldsymbol{\beta}}=0$. Meanwhile, the boundary conditions on $\left(\beta_{\alpha}, w\right)$ lead to $\left.\tilde{\beta}_{\alpha}\right|_{\partial \Omega}=\left.\tilde{w}\right|_{\partial \Omega}=\left.\nabla \tilde{w}\right|_{\partial \Omega}=0$. The last equation implies, for smooth domain, $\partial \tilde{w} /\left.\partial n\right|_{\partial \Omega}=0$.

On the other hand, by (4.11), $\zeta^{2}\|\ddot{\boldsymbol{\beta}}\|_{0} \rightarrow 0$. Thus, the first two equations of (4.4) yield $E A_{\alpha}(\boldsymbol{\beta})-\lambda \zeta^{-2}\left(w,_{\alpha}-\beta_{\alpha}\right) \rightarrow 0$. That means $\lambda \zeta^{-2}\left(w_{\alpha}-\beta_{\alpha}\right) \rightarrow E A_{\alpha}(\tilde{\boldsymbol{\beta}})=E A_{\alpha}(\nabla \tilde{w})$. Then the third equation of $(4.4)$ gives $\rho_{0} \ddot{\tilde{w}}-E \nabla \cdot(\mathbf{A}(\nabla \tilde{w}))=\rho_{0} \ddot{\tilde{w}}+D_{0} \nabla^{4} \tilde{w}=g$. The last equality can be easily verified with the definition of the operator $\mathbf{A}$ and considered in the weak sense. Similar statement for the variational equation is straightforward.

Remark 4.2. The generic constant $C$ involved in the inequalities derived in Theorem 3.4 is thickness dependent. So the boundedness of $\beta$ and $w$ in higher spaces may not be uniform with respect to the thickness. It is worth noting that the boundary layer is found for static problems of R-M plate [1]. With clamped boundary conditions, as in our case, $\partial^{3} \beta / \partial n^{3}=$ $O\left(\zeta^{-1}\right)$ near the boundary, that is, $\|\beta\|_{3}=O\left(\zeta^{-1 / 2}\right)$. The boundary layer is expected for the dynamics problem too, which warrants further investigation. Since $\tilde{\boldsymbol{\beta}}=\nabla \tilde{\boldsymbol{w}}$, it is not optimistic that we can have the convergence of $w \rightarrow \tilde{w}$ in the sense of $H^{4}$, although the corresponding K-L plate can have a strong solution $\tilde{w}$ in $H^{4}$.

\section{Summary}

Existence and uniqueness of $H^{1}$ solution of R-M plate for elastodynamics with homogeneous Dirichlet boundary conditions and general initial conditions were proved. The solution with smoother data was further investigated and proved to be in $H^{2}$. Furthermore, with higher smoothness of data and certain compatibility requirements satisfied, higher regularity of the solution was proved. With the introduction of mass scaling, along with the load scaling, the $H^{2}$ solution of R-M plate was proved to approach the $H^{2}$ weak solution of K-L plate when the thickness approaches zero. 


\section{Acknowledgments}

The author is greatly indebted to Professor F. Brezzi for the fruitful discussion about the dynamics of Reissner-Mindlin plate and the mass scaling. The author is also pleased to acknowledge the referee's helpful comments.

\section{References}

[1] D. N. Arnold and R. S. Falk, Edge effect in the Reissner-Mindlin plate theory, Analytic and Computational Models of Shells (A. K. Noor, T. Belytschko, and J. C. Simo, eds.), A.S.M.E., New York, 1989, pp. 71-90.

[2] _ A uniformly accurate finite element method for the Reissner-Mindlin plate, SIAM J. Numer. Anal. 26 (1989), no. 6, 1276-1290.

[3] K. J. Bathe and F. Brezzi, On the convergence of a four-node plate bending element based on Mindlin-Reissner plate theory and a mixed interpolation, The Mathematics of Finite Elements and Applications V (Uxbridge, 1984), Academic Press, London, 1985, pp. 491-503.

[4] F. Brezzi, On scaling of Reissner-Mindlin plate, Private communication (2003).

[5] F. Brezzi and M. Fortin, Numerical approximation of Mindlin-Reissner plates, Math. Comp. 47 (1986), no. 175, 151-158.

[6] P. G. Ciarlet, Mathematical Elasticity. Vol. I, Three-Dimensional Elasticity, Elsevier Science Publishing, New York, 1988.

[7] G. Duvaut and J.-L. Lions, Inequalities in Mechanics and Physics, Springer-Verlag, Berlin, 1976.

[8] L. C. Evans, Partial Differential Equations, Graduate Studies in Mathematics, vol. 19, American Mathematical Society, Rhode Island, 1998.

[9] K. M. Liew, C. M. Wang, Y. Xiang, and S. Kitipornchai, Vibration of Mindlin Plates, Programming the P-Ersion Ritz Method, Elsevier, Oxford, 1998.

[10] J. L. Lions and E. Magenes, Non-Homogeneous Boundary Value Problems and Applications. Vol. I, Springer-Verlag, New York, 1972.

[11] - Non-Homogeneous Boundary Value Problems and Applications. Vol. II, SpringerVerlag, New York, 1972.

[12] M. Lyly, R. Stenberg, and T. Vihinen, A stable bilinear element for the Reissner-Mindlin plate model, Comput. Methods Appl. Mech. Engrg. 110 (1993), no. 3-4, 343-357.

[13] R. D. Mindlin, Influence of rotatory inertia and shear on flexural motions of isotropic elastic plates, J. Appl. Mech. 18 (1951), 31-38.

[14] E. Reissner, The effect of transverse shear deformation on the bending of elastic plates, J. Appl. Mech. 12 (1945), A-69-A-77.

[15] _ On bending of elastic plates, Quart. Appl. Math. 5 (1947), 55-68.

[16] M. Suri, I. Babuška, and C. Schwab, Locking effects in the finite element approximation of plate models, Math. Comp. 64 (1995), no. 210, 461-482.

[17] R. Temam, Navier-Stokes Equations, 3rd ed., Theory and Numerical Analysis, Elsevier, Amsterdam, 1984.

[18] Z. Zhang and S. Zhang, Wilson's element for the Reissner-Mindlin plate, Comput. Methods Appl. Mech. Engrg. 113 (1994), no. 1-2, 55-65.

Shen Rong Wu: Research and Advanced Engineering, Ford Motor Company, 2101 Village Road, SRL-MD 2115, Dearborn, MI 48124, USA

E-mail address: swu@ford.com 


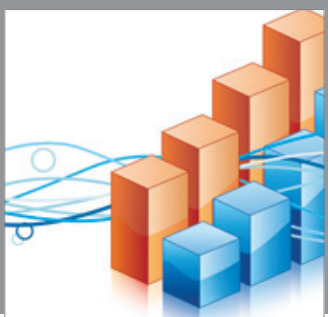

Advances in

Operations Research

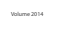

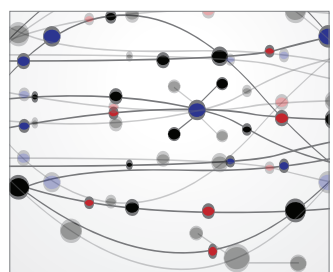

\section{The Scientific} World Journal
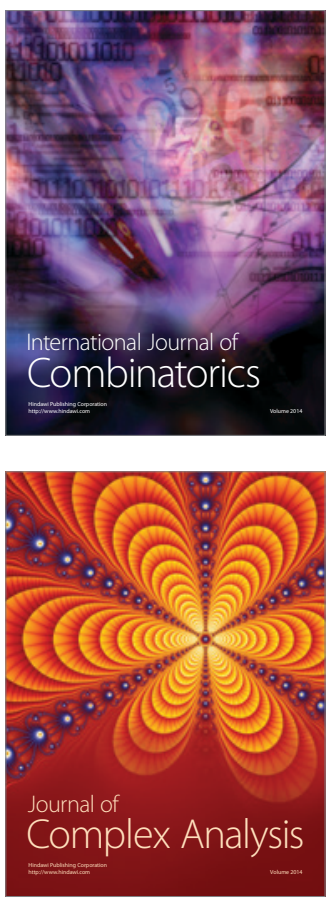

International Journal of

Mathematics and

Mathematical

Sciences
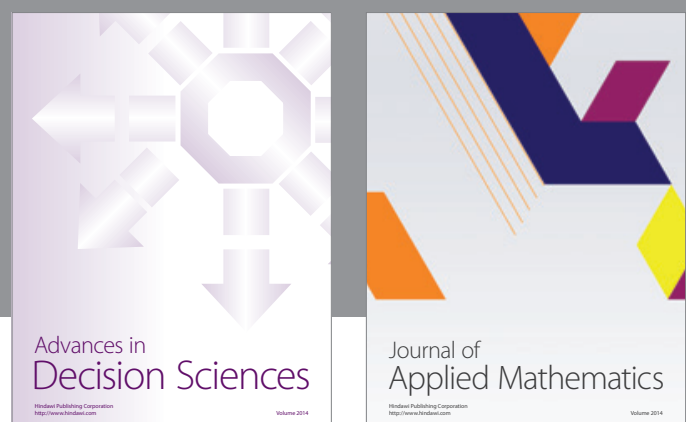

Journal of

Applied Mathematics
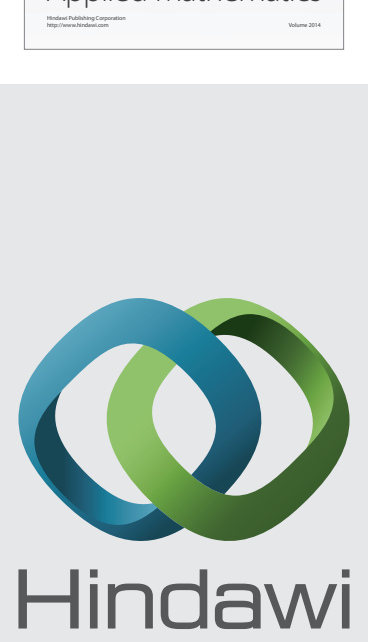

Submit your manuscripts at http://www.hindawi.com
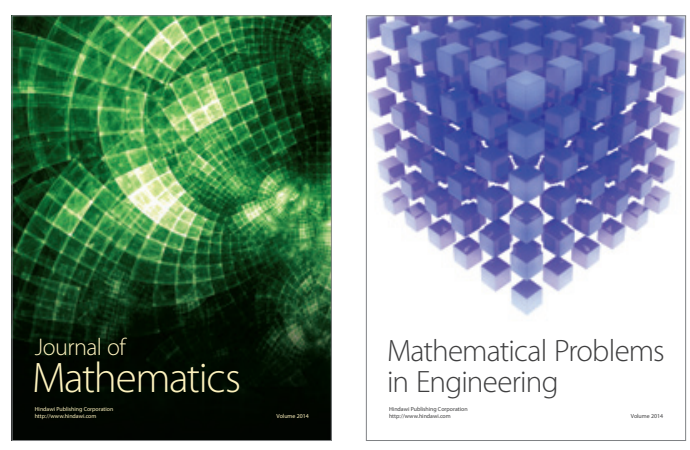

Mathematical Problems in Engineering
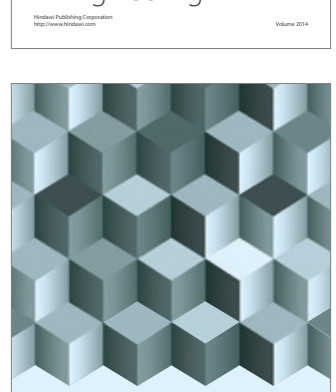

Journal of

Function Spaces
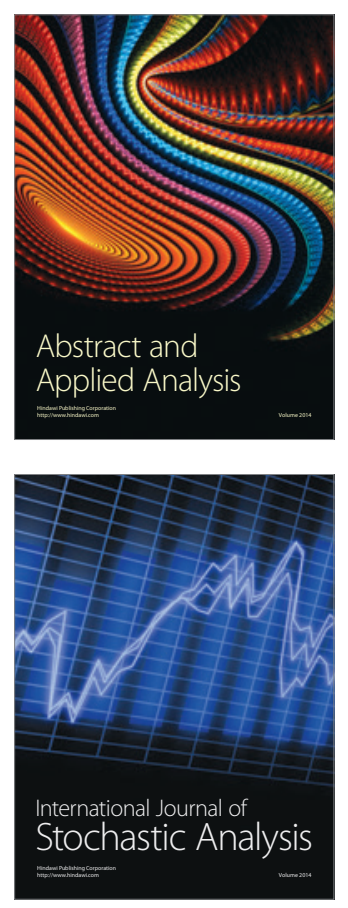

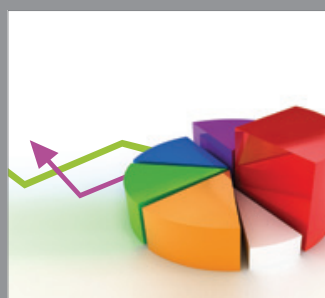

ournal of

Probability and Statistics

Promensencen
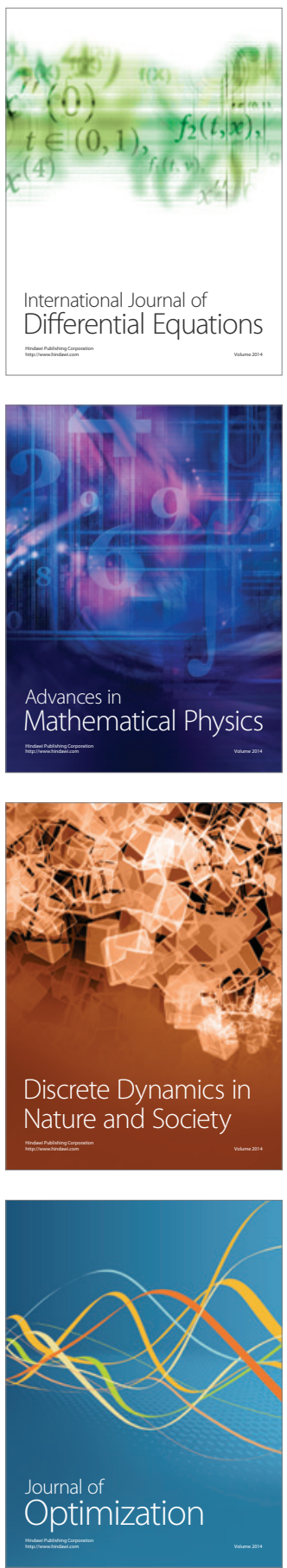